\title{
Determining Optimal Replacement Policy with an Availability Constraint via Genetic Algorithms
}

\author{
Shengliang Zong, ${ }^{1}$ Guorong Chai, ${ }^{1}$ and Yana $\mathrm{Su}^{2}$ \\ ${ }^{1}$ School of Management, Lanzhou University, Lanzhou, Gansu 730000, China \\ ${ }^{2}$ College of Economics and Management, Lanzhou Institute of Technology, Lanzhou 730050, China \\ Correspondence should be addressed to Shengliang Zong; zongshl03@gmail.com
}

Received 22 April 2016; Revised 23 November 2016; Accepted 15 December 2016; Published 18 January 2017

Academic Editor: Marco Mussetta

Copyright (c) 2017 Shengliang Zong et al. This is an open access article distributed under the Creative Commons Attribution License, which permits unrestricted use, distribution, and reproduction in any medium, provided the original work is properly cited.

\begin{abstract}
We develop a model and a genetic algorithm for determining an optimal replacement policy for power equipment subject to Poisson shocks. If the time interval of two consecutive shocks is less than a threshold value, the failed equipment can be repaired. We assume that the operating time after repair is stochastically nonincreasing and the repair time is exponentially distributed with a geometric increasing mean. Our objective is to minimize the expected average cost under an availability requirement. Based on this average cost function, we propose the genetic algorithm to locate the optimal replacement policy $N$ to minimize the average cost rate. The results show that the GA is effective and efficient in finding the optimal solutions. The availability of equipment has significance effect on the optimal replacement policy. Many practical systems fit the model developed in this paper.
\end{abstract}

\section{Introduction}

Power equipment such as a wind power generation system is operating in a dynamic and uncertain environment. Such equipment may fail due to random events such as sand/rain storms, ice rain, heavy snow, and extreme temperatures. The service interruptions caused by the equipment failure will affect the company's performance in terms of revenue and customer satisfaction. For example, during January 10 to February 5, 2008, an unexpected strong East Asian winter monsoon occurred with extremely low temperatures, blizzard conditions, and freezing rain in southern China. Due to its unusual persistence and intensity, this extreme event caused extensive damage and general disruption over southern China. Most of the infrastructure damages were attributed to freezing rain, which led to a huge amount of broken power equipment, electric power transmission lines, and chaotic traffic conditions for southern China. The disastrous heavy snow caused direct economic losses of 53.8 billion RMB [1,2].

In practice, the power equipment may be interrupted by random shocks, which represent severe weather conditions such as thunder storm and freezing rain or operational contingencies such as short circuit. When a shock arrives, it will cause a random amount of damage to the system. When the accumulated amount of shock damage exceeds a specified threshold, the system will fail. Therefore, it is necessary to consider the maintenance problem under random shocks. Chen et al. [3] investigated the impact of the voltage imbalance on the reliability of power distribution systems. Kiureghian [4] considered the problem of distribution of electric substation equipment suffering from earthquake.

Recently, shock models were utilized to model the operating time. Conceptually, a system fails due to the shock effect on the system. While most of existing shock models were based on the accumulated or extreme damage causing a system failure. Li [5] first introduced the $\delta$-shock model to avoid measuring amount of damage which may not be easy in many situations. Although the shock's damage amount is difficult to measure, its arrival time can be accurately recorded. Therefore, the $\delta$-shock model focuses mainly on the frequency of shocks rather than their accumulated damage amount of shocks. In a $\delta$-shock model, a shock causes a system failure which also called a deadly shock if the time interval between two successive shocks is smaller than 
a critical value $\delta$. The threshold $\delta$ is usually a constant. In this paper, we adopt a general $\delta$-shock model by letting $\delta$ be an exponentially distributed random variable with parameter varying with number of repairs [6]. The power equipment after repair can be more fragile and more prone to fail again. As a result, the threshold of a deadly shock is increasing with $n$, which is the number of repairs.

There are extensive studies on the maintenance problems for the system with operating and repair stages. This is mainly because some classical assumptions are not realistic in modeling the real systems. These assumptions include repaired system becoming "as good as new" and a dialed system being replaced by a new one immediately. Barlow and Proschan [7] introduced an imperfect repair model, where the repair is prefect with probability $p$ and minimal with probability $1-p$. We also consider the nonzero repair and replacement times in contrast to a classical assumption that repairs are instantaneous. In most practical situations, to reflect the aging process of the system, the consecutive repair times are assumed to become longer and longer till the system is replaced with a new one according to some replacement rule. The geometric processes (GP) method, with its practical advantages in characterizing the whole operation process of the components in a system [8], gains worldwide scholar's attention in recent years, and many researches have been carried out based on GP in different application domains. Lam [9] first introduced GP to study the maintenance problem for the deteriorating system. Finkelstein [10] generalized Lam's work by scale transformation. Most of the studies based on GP denote a good applicability in the related fields, but their assumption that the property of the component after premaintenance is as good as new is not fully compatible with the electrical power equipment power [11].

Ensuring system function and life are two very important maintenance goals [12]. Li et al. [13] designed an age-related dynamical model to create a timely maintenance schedule that extends the life of the equipment and improves its efficiency. Preventive maintenance also needs to consider how to reduce costs [14]. Castanier et al. [15] proposed a parametric maintenance decision framework to coordinate inspection and replacement of the two components and to minimize the long-run maintenance cost of the system. Lin and Huang [16] used a nonhomogeneous Poisson process with a power law failure intensity to describe the deterioration of a repairable system and to minimize the expected total cost per unit time to determine the optimal nonperiodic maintenance schedule. As we all know, the goal of the maintenance decision is not only to minimize costs. We adopt the replacement policy by satisfying certain requirements of equipment availability. Farag and Al-Baiyat [17] consider two major problems of power system design optimization. One is to maximize the reliability under the cost constraint and the other is to minimize the cost under the reliability constraint. Levitin and Lisnianski [18] studied the optimization problem of the power system containing a bridge substructure in which redundant elements are included in order to provide a desired level of reliability. There are more research works on power system structure optimization under reliability constraints, for example, Lisnianski et al. [19] and Bevrani et al. [20].

Existing research goal is to minimize the total cost or the long-run maintenance cost of the system, and the objective function mainly is linear. In this paper, the objective function is an integer nonlinear programming, and constraints are an integer nonlinear inequality. In order to try to solve this kind of problem in an appropriate way, a method based on genetic algorithms (GA) is proposed to explore the optimality of this problem. GA is heuristic search techniques, which are full of promise to deal with complex optimization problems [21]. GA can also solve optimization problems possessing arbitrary degrees of nonlinearity, discontinuity, and stochasticity. Levitin and Lisnianski [22] used GA to minimize the sum of costs of system modernization actions over the study period while satisfying reliability constraints at each stage. Abdulwhab et al. [23] used GA optimization technique to maximize the overall system reliability for a specified future time period in which a number of generating units are to be removed from service for preventive maintenance.

In this paper, we propose a $\delta$-shock model, which will be helpful for practitioners to find the appropriate machine maintenance and replacement policies, for electrical power equipment. We assume that the shocks will arrive according to a renewal process with an exponential distributed interval time of shocks and that the threshold of a deadly shock forms a geometrical increase. Furthermore, the distribution function of the threshold follows exponential distribution. The assumptions and analysis of the model are introduced in Section 2. Section 3 presents an optimization model which is to minimize the long-run average cost per unit time subject to some availability constraints. The GA is introduced in Section 4. Finally, Section 5 provides a case study using our approach.

\section{Model and Analysis}

2.1. Model Assumptions. To construct the $\delta$-shock model, we first introduce the definition of GP as follows.

Definition 1. A stochastic process $\left\{\xi_{n}, n=1,2, \ldots\right\}$ is called a geometric increasing (or decreasing) process, if there is a real number $0<a \leq 1$ (or $a \geq 1$ ), such that $\left\{a^{n-1} \xi_{n}, n=1,2, \ldots\right\}$ forms a renewal process. $a$ is called the ratio of the GP.

Moreover, let $E\left(\xi_{1}\right)=\tau$ and $\operatorname{Var}\left(\xi_{1}\right)=\sigma^{2}$. Then, we have

$$
\begin{gathered}
E\left(\xi_{n}\right)=\frac{\tau}{a^{n-1}}, \\
\operatorname{Var}\left(\xi_{n}\right)=\frac{\sigma^{2}}{a^{2(n-1)}} .
\end{gathered}
$$

Therefore, $a, \tau$, and $\sigma^{2}$ are three parameters for a GP.

We then propose a repair model for electrical power equipment under availability constraints based on the following assumptions.

(I) At the beginning, a new system is installed. Whenever the system fails, it will be immediately either repaired or replaced by an identical system. 
(II) The system in operation is subject to environment shocks which arrive according to a Poisson process with rate $\lambda_{1}$ or $E X_{n}=1 / \lambda_{1}$. Let $X_{n}(n=1,2, \ldots)$ be the time interval between the $(n-1)$ th and the $n$th shocks. Then $X_{n}$ is assumed to be exponentially distributed with the distribution function denoted by $F(x)$. Let $\delta_{n}$ be another exponentially distributed random variable associated with $X_{n}$. We assume that the sequence $\left\{\delta_{n}, n=1,2, \ldots\right\}$ forms an increasing geometric process with $0<a \leq 1$. Then, $\delta_{n}$ has cumulative distribution function $Q\left(a^{n-1} x\right)$, where $Q(x)$ is the cumulative distribution function of $\delta_{1}$ which is a brand new system's threshold of the shock model. Assume that $E \delta_{1}=1 / \lambda_{2} .\left\{X_{n}, \delta_{n}\right\}$ follows a $\delta$ shock model if the system fails at $n$th shock which satisfies $X_{n} \leq \delta_{n}$, and then the life time or equivalently the operating time is the sum of all $X_{n}$ until the one satisfying the above condition. Further, we assume that $X_{n}$ is independent of $\delta_{n}$.

(III) Let $T_{n}$ be the operating time between the $(n-1)$ th and $n$th repairs. $\left\{T_{n}, n=1,2, \ldots\right\}$ is a sequence of stochastically decreasing random variables.

(IV) Let $Y_{n}$ denote the repair time after the $n$th failure. Then the distribution function of $Y_{n}$ is $G\left(b^{n-1} y\right)$, where $0<$ $b \leq 1$ is a constant. Let $E\left(Y_{n}\right)$ denote the expected repair time after the $n$th failure. Assume that $E(Y)=\mu>0$.

(V) $T_{n}$ and $Y_{n}, n=1,2, \ldots$, are independent.

(VI) The reward and cost structure is assumed as follows: the reward rate when the system is operating is $r$. The repair cost rate of the system is $c$. The replacement cost consists of a fixed replacement cost $R$ and variable cost $v=r_{p} Z$, where $r_{p}$ is the variable cost rate and $Z$ is the replacement time with a mean of $E(Z)=t$.

(VII) The $\mathrm{N}$-replacement policy is adopted. Under such a policy, the system will be replaced with a new and identical one at the $N$ th failure.

(VIII) The equipment must meet the minimum availability requirements. Let $p$ represent the availability of the equipment. In other words, the proportion of the nonproductive time, for example, repair or replacement, should not be too high.

2.2. Maintenance Cost. Since $Y_{n}, n=1,2, \ldots$, forms an increasing GP with ratio $0<b \leq 1$, we have

$$
E\left(Y_{n}\right)=\frac{\mu}{b^{n-1}} .
$$

Thus, the maintenance cost function is shown as

$$
\mathrm{MC}=\sum_{n=1}^{x-1} E\left(c Y_{n}\right)=\sum_{n=1}^{x-1} \frac{c \mu}{b^{n-1}} .
$$

2.3. Operating Revenue. Let $E\left(T_{n}\right)$ be the expected operating time of the system between the $(n-1)$ th and $n$th repairs, and let $l_{n i}$ be the interval time between the $(i-1)$ th and $i$ th shock following the $(n-1)$ th repair. Then the number of shocks experienced until the next failure can be written as

$$
\begin{aligned}
M_{n} & =\min \left\{m \mid l_{n 1}>a^{n-1} \delta_{1}, \ldots, l_{n(m-1)}>a^{n-1} \delta_{1}, l_{n m}\right. \\
& \left.<a^{n-1} \delta_{1}\right\} .
\end{aligned}
$$

We have

$$
T_{n}=\sum_{i=1}^{M_{n}} l_{n i}
$$

Here $M_{n}$ follows a geometric distribution. That is

$$
P\left(M_{n}=k\right)=q_{n}^{k-1} p_{n}, \quad k=1,2, \ldots,
$$

where $p_{n}=P$ (a shock following the $(n-1)$ th repair is a deadly shock) and $q_{n}=1-p_{n}$.

Therefore, we have $E M_{n}=1 / p_{n}$. As $M_{n}$ is a stopping time with respect to the random sequence $\left\{l_{n i}, i=1,2, \ldots\right\}$, which are independent identically distributed random variables. According to Wald's equation [24], we have

$$
E\left(T_{n}\right)=E\left(\sum_{i=1}^{M_{n}} l_{n i}\right)=E l_{n 1} E M_{n}=\frac{E l_{n 1}}{p_{n}} .
$$

Based on Assumption (II), and the fact that $F(x)$ and $Q(x)$ are exponential distributions, we have

$$
\begin{gathered}
F(x)=1-e^{-\lambda_{1} x}, \quad x \geq 0, \\
Q\left(a^{n-1} x\right)=1-e^{-a^{n-1} \lambda_{2} x}, \quad x \geq 0 .
\end{gathered}
$$

Furthermore, as $l_{n i}$ and $\delta_{n}$ are independent and have the marginal exponential distributions with means of $1 / \lambda_{1}$ and $1 / a^{n-1} \lambda_{2}$, therefore, we obtain

$$
\begin{aligned}
p_{n} & =P\left(l_{n i}<\delta_{n}\right)=\int_{0}^{\infty} F(x) d Q(x) \\
& =\int_{0}^{\infty}\left(1-e^{-\lambda_{1} x}\right) d\left(1-e^{-a^{n-1} \lambda_{2} x}\right) \\
& =\frac{\lambda_{1}}{\lambda_{1}+a^{n-1} \lambda_{2}}, \\
E l_{n 1} & =p_{n}=\int_{0}^{\infty} x d F(x)=\int_{0}^{\infty} x d\left(1-e^{-\lambda_{1} x}\right)=\frac{1}{\lambda_{1}} .
\end{aligned}
$$

Then

$$
\zeta_{n}=E\left(T_{n}\right)=\frac{E l_{n 1}}{p_{n}}=\frac{\lambda_{1}+a^{n-1} \lambda_{2}}{\lambda_{1}^{2}} .
$$

Consequently,

$$
E\left(\sum_{n=1}^{x} T_{n}\right)=\sum_{n=1}^{x} E\left(T_{n}\right)=\sum_{n=1}^{x} \frac{\lambda_{1}+a^{n-1} \lambda_{2}}{\lambda_{1}^{2}} .
$$

Then, the operating revenue of the system, denoted by $\mathrm{OR}$, is given by

$$
\mathrm{OR}=\sum_{n=1}^{x} r E\left(T_{n}\right)=r \sum_{n=1}^{x} \frac{\lambda_{1}+a^{n-1} \lambda_{2}}{\lambda_{1}^{2}} .
$$

2.4. Replacement Cost. Based on Assumption (VI), the replacement cost function, denoted by $\mathrm{RC}$, can be written as

$$
\mathrm{RC}=E(R+v)=R+r_{p} t .
$$


2.5. Long-Run Average Cost. Let $C(x)$ denote the long-run average cost per unit time of the system. According to renewal reward theorem [24]

$$
C(x)=\frac{\text { Expected cost incurred in a cycle }}{\text { Expected length in a cycle }} .
$$

Let $W$ be the expected length of a renewal cycle of the system under replacement policy $N$. Thus, we have

$$
W=E\left(\sum_{n=1}^{x} T_{n}+\sum_{n=1}^{x-1} Y_{n}+Z\right)
$$

Let TC be the total expected cost of the renewal cycle of the system under replacement policy $N$. Then,

$$
\mathrm{TC}=\mathrm{MC}-\mathrm{OR}+\mathrm{RC}
$$

Consequently,

$$
\begin{aligned}
& C(x)=\frac{\mathrm{TC}}{W} \\
& =\frac{c \sum_{n=1}^{x-1}\left(\mu / b^{n-1}\right)-r \sum_{n=1}^{x}\left(\left(\lambda_{1}+a^{n-1} \lambda_{2}\right) / \lambda_{1}^{2}\right)+R+r_{p} t}{\sum_{n=1}^{x}\left(\left(\lambda_{1}+a^{n-1} \lambda_{2}\right) / \lambda_{1}^{2}\right)+\sum_{n=1}^{x-1}\left(\mu / b^{n-1}\right)+t} .
\end{aligned}
$$

\section{Optimization}

3.1. System Availability. Using (13) and (17), the availability function of the system can be written as

$$
\begin{aligned}
& p(x) \\
& =\frac{\sum_{n=1}^{x}\left(\left(\lambda_{1}+a^{n-1} \lambda_{2}\right) / \lambda_{1}^{2}\right)}{\sum_{n=1}^{x}\left(\left(\lambda_{1}+a^{n-1} \lambda_{2}\right) / \lambda_{1}^{2}\right)+\sum_{n=1}^{x-1}\left(\mu / b^{n-1}\right)+t}, \\
& x \in N .
\end{aligned}
$$

3.2. Minimizing the Average Cost. The optimal policy can be found by minimizing the cost of each unit time subject to a minimum level of system availability. The optimization problem is

$$
\begin{aligned}
\min & C(x)=\frac{c \sum_{n=1}^{x-1}\left(\mu / b^{n-1}\right)-r \sum_{n=1}^{x}\left(\left(\lambda_{1}+a^{n-1} \lambda_{2}\right) / \lambda_{1}^{2}\right)+R+r_{p} t}{\sum_{n=1}^{x}\left(\left(\lambda_{1}+a^{n-1} \lambda_{2}\right) / \lambda_{1}^{2}\right)+\sum_{n=1}^{x-1}\left(\mu / b^{n-1}\right)+t} \\
\text { subject to } & \frac{\sum_{n=1}^{x}\left(\left(\lambda_{1}+a^{n-1} \lambda_{2}\right) / \lambda_{1}^{2}\right)}{\sum_{n=1}^{x}\left(\left(\lambda_{1}+a^{n-1} \lambda_{2}\right) / \lambda_{1}^{2}\right)+\sum_{n=1}^{x-1}\left(\mu / b^{n-1}\right)+t} \geq p . \\
& x \in N \\
& 0 \leq a \leq 1,0 \leq b \leq 1 .
\end{aligned}
$$

Thus, we can see that the optimization problem is an integer nonlinear programming problem. The analytical solution is not effective to solve this optimization problem, because the objective function $C(x)$ is a nonconvex and extremely complex function. Several classical computational techniques, for example, branch and bound technique, cutting planes technique, implicit enumeration, and out approximation, which are reasonably efficient, have been proposed in literature for solving integer nonlinear programming problems [2527]. These techniques are applicable to a particular class of problem. In the case of nonconvex problems these techniques may cut-off the global optimal.

\section{Genetic Algorithms}

In the last two decades, a number of stochastic algorithms have been proposed for solving the integer nonlinear programming problems. Genetic algorithms have been proved to be a useful tool in solving global optimization problems [28]. GA is general purpose population based stochastic search techniques which mimic the principles of natural selection and genetics. It can be implemented easily with the computer programming. In particular, GA is more effective to solve the large scale, real life, discrete, and continuous optimization problems without making unrealistic assumptions and approximations. Recently, GA has been proposed to solve integer programming problems by Soltani and Shafiei [29] and Wong et al. [30]. Applying GA to solve nonlinear mixed integer optimization problems was reported by Pal et al. [31] and Deep et al. [32].

Our objective function is an integer nonlinear programming and constraints are an integer nonlinear inequality. For solving such a problem, we have developed a real coded GA for integer variables.

It is generally accepted that any GA consists of the following basic components.

Step 1 (set the parameters). The parameters of the GA in our problem include population size, probability of crossover 
(pc), probability of mutation (pm), and stopping conditions. The population size should be neither too large nor too small. We set the parameters as follows:

Population size $=10, \mathrm{pc}=0.90, \mathrm{pm}=0.10$, stall generations as the stopping conditions.

Step 2 (generate initial populations). An initial population of size $n$ is randomly generated. Any general procedure to get them can be applied. Nevertheless, in the implementation, we prefer to build the initial population with feasible chromosomes. This device guarantees that, upon termination, the method will provide a bilevel feasible solution. A positive integer is randomly generated according to the nature of the problem. It follows the next law $l=$ ceil $(\min x(1)+(\max x$ $(1)-\min x(1)) *$ rand $($ Size, 1$))$.

Step 3 (calculate the fitness values). Each chromosome in the population is evaluated by creating the estimated value for each coefficient in (19). The fitness value of each chromosome is then obtained from (20). Let, $f(i)$ be the fitness value for each chromosome and the total fitness value of the populations is $T$, where $T=\operatorname{sum} f(i)$.

Step 4 (perform selection). The roulette wheel selection mechanism is the selection strategy chosen in our paper. Each time, a single chromosome is selected from the current generation to create a new generation. The selection process is as follows. Each time, a random number $h$ is generated from the range $[0,1]$. Let $p r_{(i)}$ be the weight of the $i$ th chromosome in the whole populations, where $p r_{(i)}=f(i) / \operatorname{sum} f(i)$. If $h<p r_{(i)}$, then this chromosome is selected. Otherwise, the $j$ th chromosome is selected if $\operatorname{pr}_{(j-1)}<h<p r_{(j)}(i<j)$. This selection process is continued until the new population has been created. The chromosome which has the higher fitness value tends to be picked out to the next generation.

Step 5 (perform crossover). Crossover is the key to the power of genetic algorithms. The purpose of the crossover is to generate offspring which endorses them with the common character of their parents. Calvete et al. [33] proposed two types of crossover methods. One is called "variable-tovariable" crossover and the other is called "basis-to-basis" crossover. Lin [34] used the one-point crossover method, which randomly selects one cut-point and exchanges the right parts of two randomly selected parents in the population to generate offspring. The crossover method of this paper uses also the one-point method.

Step 6 (perform mutation). Mutation is an operator that acts on various chromosomes by randomly exchanging one of its indices. Mutation resets a selected position in a chromosome to a randomly generated real number in $[0,1]$. The mutation rate is usually a very small value; on average, $0.3 \%$ of the total populations undergo mutation [34]. The mutation method used here is the midvalue method, where the mutation of chromosome took place in the middle of the population. The new chromosome contains the indices of the new basis.
TABLE 1: Value of the parameters.

\begin{tabular}{lcccccccccc}
\hline Parameters & $a$ & $b$ & $c$ & $\mu$ & $R$ & $r_{p}$ & $t$ & $\lambda_{1}$ & $\lambda_{2}$ & $p$ \\
\hline Value & 0.95 & 0.94 & 2 & 4 & 8000 & 3 & 20 & 0.005 & 0.02 & 0.98 \\
\hline
\end{tabular}

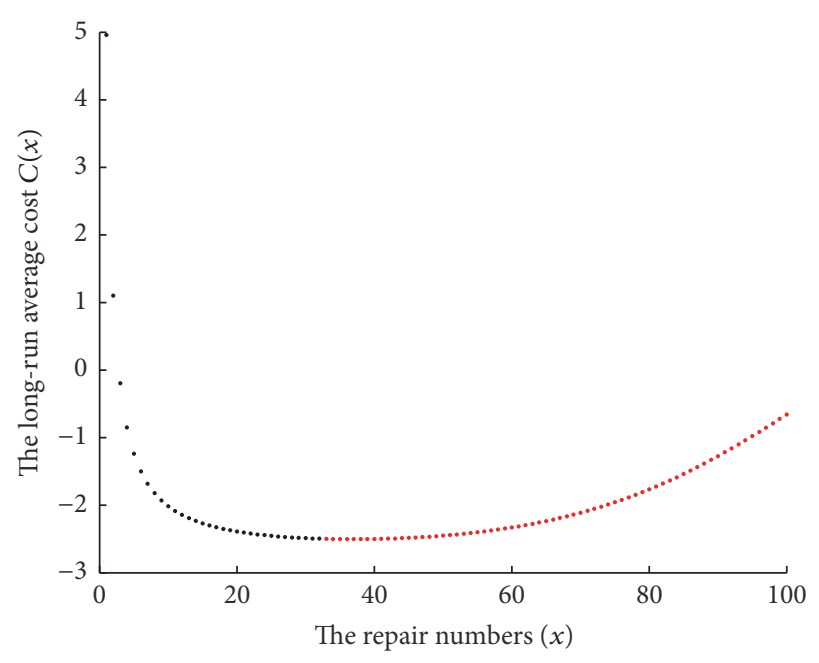

FIgURE 1: The plot of $C(x)$ against $x$.

Step 7 (return to Step 3 until the stopping condition is met). There are many stopping conditions for GA, including generations, time limit, fitness limit, stall generations, and stall time. The programs will stop until one of the stopping conditions is met. The stopping condition used here is stall generations.

\section{Empirical Results}

In this section we analyze an example using the proposed GA. All experiments were performed in MATLAB on a PC with an Intel Core i7 $2.80 \mathrm{GHz}$ and $4 \mathrm{GRAM}$. The data is simulation data which are used to further evaluate the proposed approaches. The simulation data are generated from the analytical curves. Table 1 shows the value of the parameters.

Figure 1 shows the relations between $C(x)$ and $x$. Note that the black dot meets constraints and the red dots do not. It is obvious that the best results can be obtained at $x=32$.

In order to simplify the calculations, we set the lower bound to be 0 and the upper bound to be 1000, which is a positive infinity in our problem. The appropriate parameters of GA here are set as follows.

$$
\begin{aligned}
& \text { Population size }=10 \\
& \text { Probability of crossover }=0.90 \\
& \text { Probability of mutation }=0.10 \\
& \text { Stall generations }=200
\end{aligned}
$$

The stopping condition for the GA is to reach the maximum stall generations. The simulation result is shown in Figure 2. It is interesting to observe that, in initial generations, the GA finds the configuration to minimize the cost. The 


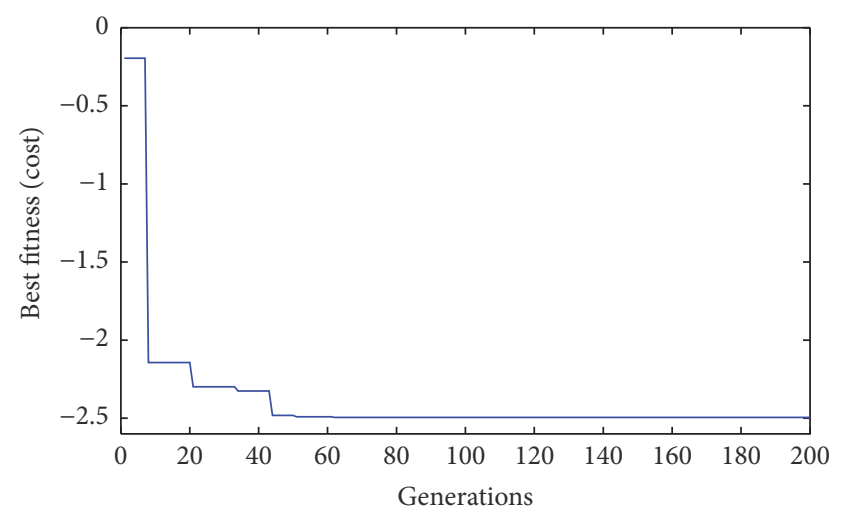

FIGURE 2: Best fitness terms evolution during the GA generations $(t=1.6719 \mathrm{~s})$.

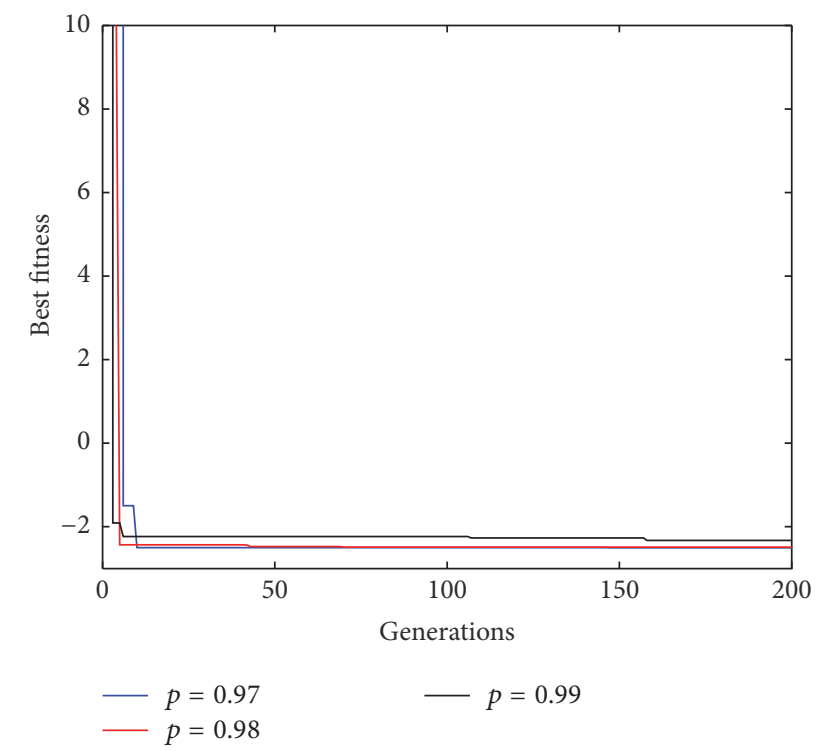

Figure 3: Best fitness terms evolution with differences $p$ during the GA generations.

fitness is suddenly reduced from -0.2 to -2.2 on the 10th generations. After 10 generations, the fitness has decreased. The global fitness is obtained at about the 45th generation. Best fitness is -2.4950 and the corresponding variable is 32 .

In addition, the GA is repeatedly executed 10 times to ensure that the global fitness can be achieved so that the local optimization can be avoided. As is shown in Table 2, it is observed that we can find the running times of different executions are different, and the 5th stop and the 7th stop do not reach the global optimal results.

Furthermore, we simulate the fitness with different constraints. Figure 3 shows that the three lines have the same trend of converging to the optimal fitness in the 10th generation. Table 3 shows that the number of repairs is decreasing and the long-run average cost per unit time is increasing in $p$. As is shown in Table 3, when the availability of the equipment is 0.97 , the best fitness is -2.5026 and the corresponding variable is 37 . Meanwhile, if the availability of the equipment
TABLE 2: Result of 10 times $(p=0.98)$.

\begin{tabular}{lcccc}
\hline & Value & Individual & Time (seconds) & $\mathrm{Y} / \mathrm{N}$ \\
\hline 1st stop & -2.4950 & 32 & 1.6250 & $\mathrm{Y}$ \\
2nd stop & -2.4950 & 32 & 1.6406 & $\mathrm{Y}$ \\
3rd stop & -2.4950 & 32 & 1.6406 & $\mathrm{Y}$ \\
4th stop & -2.4950 & 32 & 1.6406 & $\mathrm{Y}$ \\
5th stop & -2.4872 & 30 & 1.6563 & $\mathrm{~N}$ \\
6th stop & -2.4950 & 32 & 1.6719 & $\mathrm{Y}$ \\
7th stop & -2.4872 & 30 & 1.6875 & $\mathrm{~N}$ \\
8th stop & -2.4950 & 32 & 1.6719 & $\mathrm{Y}$ \\
9th stop & -2.4950 & 32 & 1.6875 & $\mathrm{Y}$ \\
10th stop & -2.4950 & 32 & 1.7031 & $\mathrm{Y}$ \\
\hline
\end{tabular}

is increasing to 0.99 , the best fitness will become -2.3261 and the corresponding variable will become 17. In practice, the availability of the equipment is decreasing with the repair times. Thus, you have to maintain more times until the equipment reached its availability, and you will obtain more benefits due to the equipment operating longer times.

\section{Conclusions}

In practice, the power equipment may be interrupted by random shocks, which represent severe weather conditions such as thunder storm and freezing rain or operational contingencies such as short circuit. When the accumulated amount of shock damage exceeds a specified threshold, the system will fail. Therefore, it is necessary to consider the maintenance problem under random shocks. In this paper, we present a nonlinear integer optimization model for power equipment to minimize the expected long-run average cost rate under availability constraints. Such a model is useful for system managers to find the optimal maintenance policies. We proposed to use the GA to solve the optimization problem. GA appears to be a strong method for solving complex global optimization problems in which gradient related methods usually give local optima. GA has global scope over the search space so they get global optima. Such an approach can be very effective in solving this type of optimization problems with respect to cost and availability. As we all know, GA is an approximate algorithm. The optimal solution may be different due to it is approximate optimal solution each time. And it may take more times until we obtain the optimal replacement policy when the scale is larger. Meanwhile, the results show that the number of repairs is decreasing and the long-run average cost per unit time is increasing with availability. In other words, you have to maintain more times before reaching the availability of the equipment, and you will obtain more benefits due to the equipment operating longer times. There is not only the availability but also the maintenance cost and effects on the optimal replacement policy. In a word, our model provides a useful quantitative tool for managers to evaluate the system performance and design an optimal maintenance policy.

In this paper, the shock model process is assumed to follow a Poisson process. Extending such a shock process into 
TABLE 3: Comparison of solutions with different $p$ values.

\begin{tabular}{ccccccc}
\hline & \multicolumn{2}{c}{$p=0.97$} & \multicolumn{2}{c}{$p=0.98$} & \multicolumn{2}{c}{$p=0.99$} \\
& Individual & Fitness & Individual & Fitness & Individual & Fitness \\
\hline \multirow{2}{*}{ Value } & 37 & -2.5026 & 32 & -2.4950 & -2.3261 \\
& 5 & $-0.3 \%$ & 0 & $0 \%$ & -15 & $6.8 \%$ \\
\hline
\end{tabular}

a more general nonhomogeneous Poisson process or renewal process can be a good topic for future research. Meanwhile, we only considered the average cost minimization problem. A possible future work also can be to maximize the availability under the cost constraints. In addition, our approach can be extended to study multiequipment of the parallel or unparallel systems.

\section{Notations}
a: $\quad$ Ratio of the GP of $\left\{\xi_{n}, n=1,2, \ldots\right\}$
$b$ : $\quad$ Ratio of the GP of $\left\{Y_{n}, n=1,2, \ldots\right\}$
$c$ : The repair cost rate of the system
$C(x)$ : Long-run average cost per unit time of the system

$f(i)$ : The fitness value for each chromosome

$F(x)$ : The distribution function of $X_{n}$

$Q(x)$ : The cumulative distribution function of $\delta_{1}$

$h$ : A random number is generated from the range $[0,1]$

$k$ : $\quad$ Same as $M_{n}$

$l_{n i}: \quad$ The interval time between the $(i-1)$ th and $i$ th shock following the $(n-1)$ th repair

$M_{n}$ : The number of shocks experienced until the next failure

$N$ : Replacement policy

OR: Operating revenue of the system

$p$ : Availability of the equipment

$\operatorname{pr}_{(i)}$ : The weight of the ith chromosome in the whole populations

$p_{n}$ : The probability of a shock following the $(n-1)$ th repair is a deadly shock

$q_{n}$ : The probability of a shock following the $(n-1)$ th repair is not a deadly shock

$r$ : The reward rate when the system operating

$r_{p}: \quad$ Variable cost rate

$R: \quad$ Fixed replacement cost

RC: Replacement cost

T: $\quad$ The total fitness value of the populations

$T_{n}$ : The operating time between the $(n-1)$ th and $n$th repairs

TC: Total expected cost of the renewal cycle of the system under replacement policy $N$

$W$ : Expected length of a renewal cycle of the system under replacement policy $N$

$x$ : Replacement times

$X_{n}$ : The time interval between the $(n-1)$ th and the $n$th shocks

$Y_{n}: \quad$ The repair time after the $n$th failure

$Z$ : $\quad$ Replacement time $\lambda_{1}$ : The rate of environment shocks which arrive

$\lambda_{2}$ : The reciprocal of expected value of $\delta_{1}$

$\sigma^{2}$ : Variance of $\xi_{1}$

$\mu$ : The expected value of $Y_{n}$

$\delta_{n}$ : Another exponentially distributed random variable associated with $X_{n}$

$\xi_{n}:$ Stochastic process

$\tau$ : Expected value of $\xi_{1}$.

\section{Competing Interests}

The authors declare that there is no conflict of interests regarding the publication of this paper.

\section{Acknowledgments}

This work was supported by the National Natural Science Foundation of China (nos. 71072070 and 71472079) and Fundamental Research Funds for the Central Universities of China (nos. 14LZUJBWZY074 and 16LZUJBWZY007).

\section{References}

[1] Y. Ding, Z. Wang, Y. Song, and J. Zhang, “The unprecedented freezing disaster in january 2008 in Southern China and its possible association with the global warming," Acta Meteorologica Sinica, vol. 22, no. 4, pp. 538-558, 2008.

[2] W. Zhou, J. C. L. Chan, W. Chen, J. Ling, J. G. Pinto, and Y. Shao, "Synoptic-scale controls of persistent low temperature and icy weather over Southern China in January 2008," Monthly Weather Review, vol. 137, no. 11, pp. 3978-3991, 2009.

[3] T. H. Chen, C. H. Yang, and T. Y. Hsieh, "Case studies of the impact of voltage imbalance on power distribution systems and equipment," in Proceedings of the 8th WSEAS International Conference on Applied Computer and Applied Computational Science, pp. 461-465, May 2009.

[4] A. D. Kiureghian, "Special issue: earthquake engineering for electric power equipment and lifeline systems-preface," Earthquake Engineering \& Structural Dynamics, vol. 36, pp. 163-165, 2007.

[5] Z. Li, "Some probability distributions on Poisson shocks and its application in city traffic," Journal of Lanzhou University, vol. 20, pp. 127-136, 1984.

[6] S. Zong, G. Chai, Z. G. Zhang, and L. Zhao, "Optimal replacement policy for a deteriorating system with increasing repair times," Applied Mathematical Modelling, vol. 37, no. 23, pp. 9768-9775, 2013.

[7] M. Brown and F. Proschan, "Imperfect repair," Journal of Applied Probability, vol. 20, no. 4, pp. 851-859, 1983. 
[8] Y. Lam and Y. L. Zhang, "A geometric-process maintenance model for a deteriorating system under a random environment," IEEE Transactions on Reliability, vol. 52, no. 1, pp. 83-89, 2003.

[9] Y. Lam, "Geometric processes and replacement problem," Acta Mathematicae Applicatae Sinica, vol. 4, no. 4, pp. 366-377, 1988.

[10] M. S. Finkelstein, "A scale model of general repair," Microelectronics Reliability, vol. 33, no. 1, pp. 41-44, 1993.

[11] Y. Li, J.-K. Wang, P. Han, and Y.-H. Han, "Geometric-processbased battery management optimizing policy for the electric bus," Mathematical Problems in Engineering, vol. 2015, Article ID 624130, 7 pages, 2015.

[12] R. Dekker, "Applications of maintenance optimization models: a review and analysis," Reliability Engineering \& System Safety, vol. 51, no. 3, pp. 229-240, 1996.

[13] D.-C. Li, C.-W. Liu, and T.-L. Chen, "Recursive operation time maximization model for the maintenance of power generation equipment," Computers \& Operations Research, vol. 39, no. 5, pp. 1117-1121, 2012.

[14] K. Linderman, K. E. McKone-Sweet, and J. C. Anderson, "An integrated systems approach to process control and maintenance," European Journal of Operational Research, vol. 164, no. 2, pp. 324-340, 2005.

[15] B. Castanier, A. Grall, and C. Bérenguer, "A condition-based maintenance policy with non-periodic inspections for a twounit series system," Reliability Engineering and System Safety, vol. 87, no. 1, pp. 109-120, 2005.

[16] Z.-L. Lin and Y.-S. Huang, "Nonperiodic preventive maintenance for repairable systems," Naval Research Logistics, vol. 57, no. 7, pp. 615-625, 2010.

[17] A. S. Farag and S. Al-Baiyat, "Optimal design of power systems under constraints of reliability and cost," Energy Conversion and Management, vol. 38, no. 7, pp. 637-645, 1997.

[18] G. Levitin and A. Lisnianski, "Structure optimization of power system with bridge topology," Electric Power Systems Research, vol. 45, no. 3, pp. 201-208, 1998.

[19] A. Lisnianski, G. Levitin, H. Ben-Haim, and D. Elmakis, "Power system structure optimization subject to reliability constraints," Electric Power Systems Research, vol. 39, no. 2, pp. 145-152, 1996.

[20] H. Bevrani, Y. Mitani, and K. Tsuji, "Robust decentralized AGC in a restructured power system," Energy Conversion and Management, vol. 45, no. 15-16, pp. 2297-2312, 2004.

[21] R. Z. Ríos-Mercado, S. Kim, and E. A. Boyd, "Efficient operation of natural gas transmission systems: a network-based heuristic for cyclic structures," Computers and Operations Research, vol. 33, no. 8, pp. 2323-2351, 2006.

[22] G. Levitin and A. Lisnianski, "Optimal multistage modernization of power system subject to reliability and capacity requirements," Electric Power Systems Research, vol. 50, no. 3, pp. 183-190, 1999.

[23] A. Abdulwhab, R. Billinton, A. A. Eldamaty, and S. O. Faried, "Maintenance scheduling optimization using a genetic algorithm (GA) with a probabilistic fitness function," Electric Power Components and Systems, vol. 32, no. 12, pp. 1239-1254, 2004.

[24] S. M. Ross, Stochastic Processes, Wiley-Interscience, New York, NY, USA, 1983.

[25] C. Still and T. Westerlund, "Solving convex MINLP optimization problems using a sequential cutting plane algorithm," Computational Optimization and Applications, vol. 34, no. 1, pp. 63-83, 2006.

[26] A. Agnetis, F. Rossi, and S. Smriglio, "An implicit enumeration scheme for the batch selection problem," Networks, vol. 44, no. 2, pp. 151-159, 2004.
[27] P. Bonami, L. T. Biegler, A. R. Conn et al., "An algorithmic framework for convex mixed integer nonlinear programs," Discrete Optimization, vol. 5, no. 2, pp. 186-204, 2008.

[28] H. Aytug and G. J. Koehler, "The effect of multiple optima on the simple GA run-time complexity," European Journal of Operational Research, vol. 178, no. 1, pp. 27-45, 2007.

[29] H. Soltani and S. Shafiei, "Heat exchanger networks retrofit with considering pressure drop by coupling genetic algorithm with LP (linear programming) and ILP (integer linear programming) methods," Energy, vol. 36, no. 5, pp. 2381-2391, 2011.

[30] C. K. Wong, I. W. H. Fung, and C. M. Tam, "Comparison of using mixed-integer programming and genetic algorithms for construction site facility layout planning," Journal of Construction Engineering and Management, vol. 136, no. 10, pp. 1116-1128, 2010.

[31] P. Pal, C. B. Das, A. Panda, and A. K. Bhunia, "An application of real-coded genetic algorithm (for mixed integer non-linear programming in an optimal two-warehouse inventory policy for deteriorating items with a linear trend in demand and a fixed planning horizon)," International Journal of Computer Mathematics, vol. 82, no. 2, pp. 163-175, 2005.

[32] K. Deep, K. P. Singh, M. L. Kansal, and C. Mohan, "A real coded genetic algorithm for solving integer and mixed integer optimization problems," Applied Mathematics and Computation, vol. 212, no. 2, pp. 505-518, 2009.

[33] H. I. Calvete, C. Galé, and P. M. Mateo, "A new approach for solving linear bilevel problems using genetic algorithms," European Journal of Operational Research, vol. 188, no. 1, pp. 1428, 2008.

[34] F.-T. Lin, "Solving the knapsack problem with imprecise weight coefficients using genetic algorithms," European Journal of Operational Research, vol. 185, no. 1, pp. 133-145, 2008. 


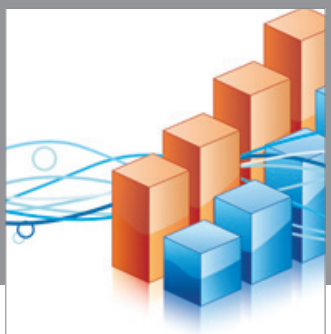

Advances in

Operations Research

vatem alat4

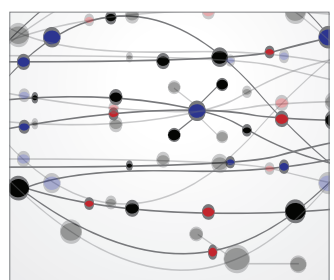

\section{The Scientific} World Journal
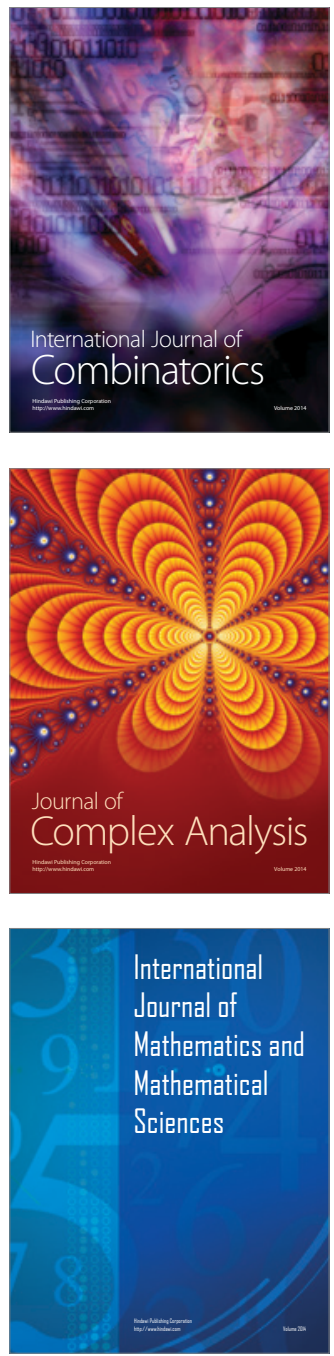
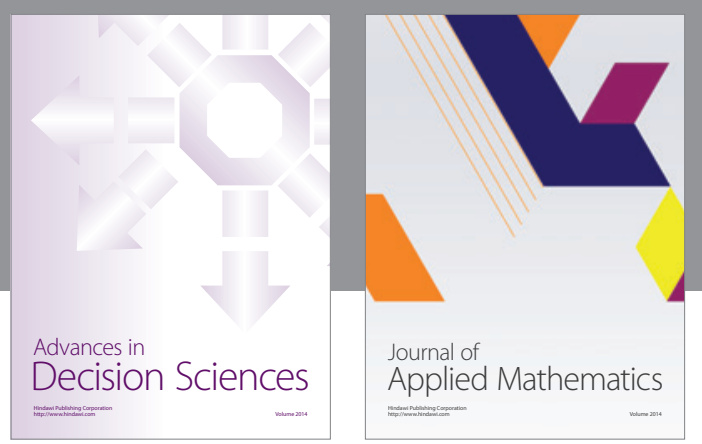

Algebra

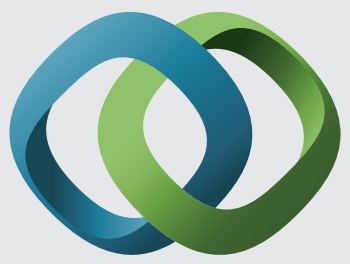

\section{Hindawi}

Submit your manuscripts at

https://www.hindawi.com
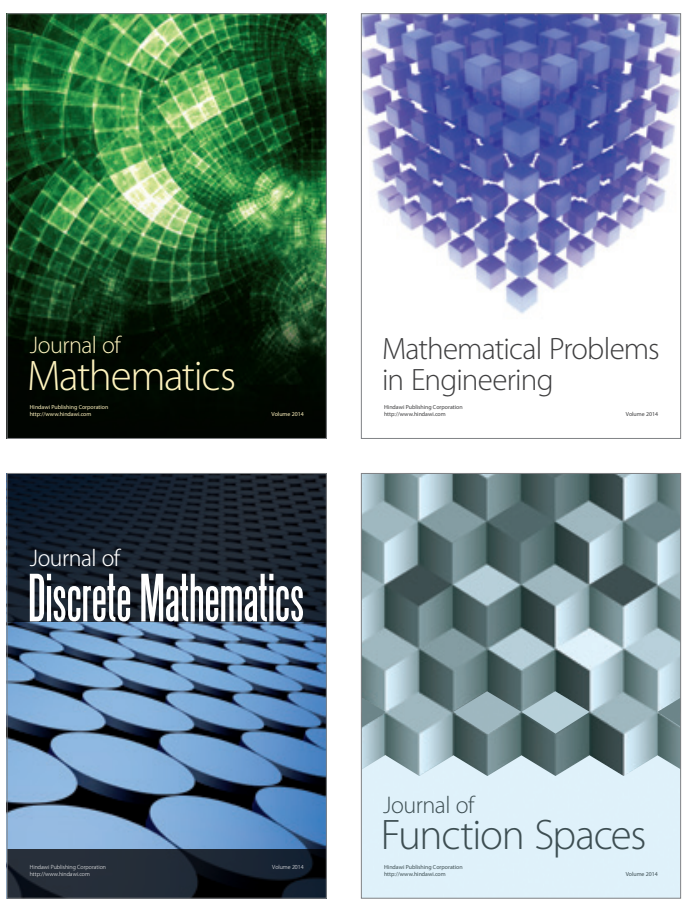

Mathematical Problems in Engineering
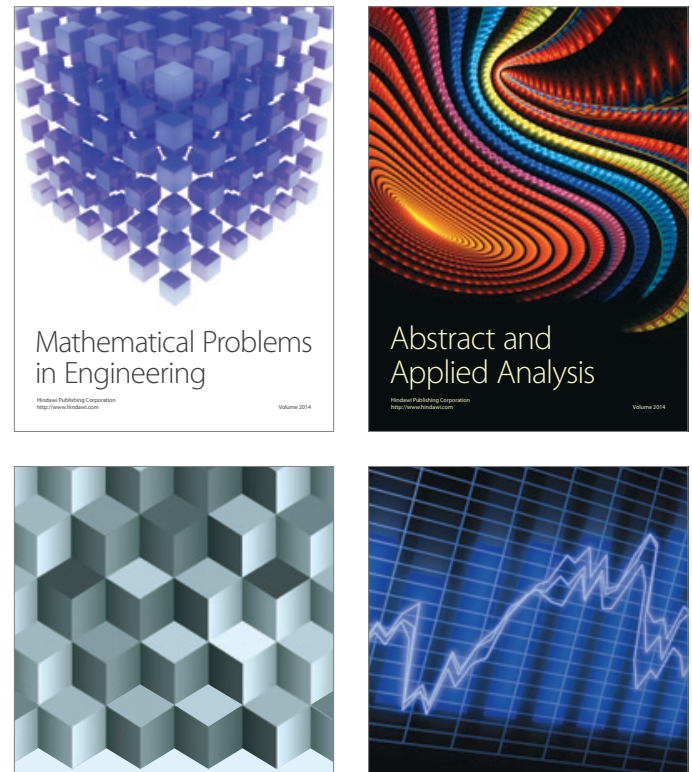

Journal of

Function Spaces

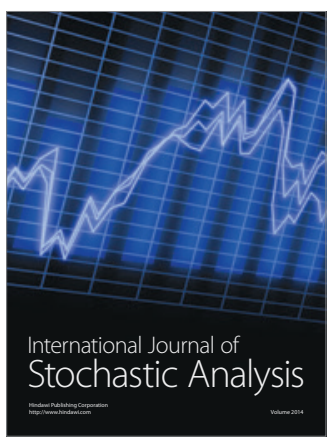

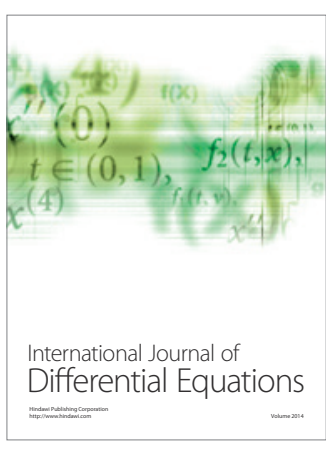
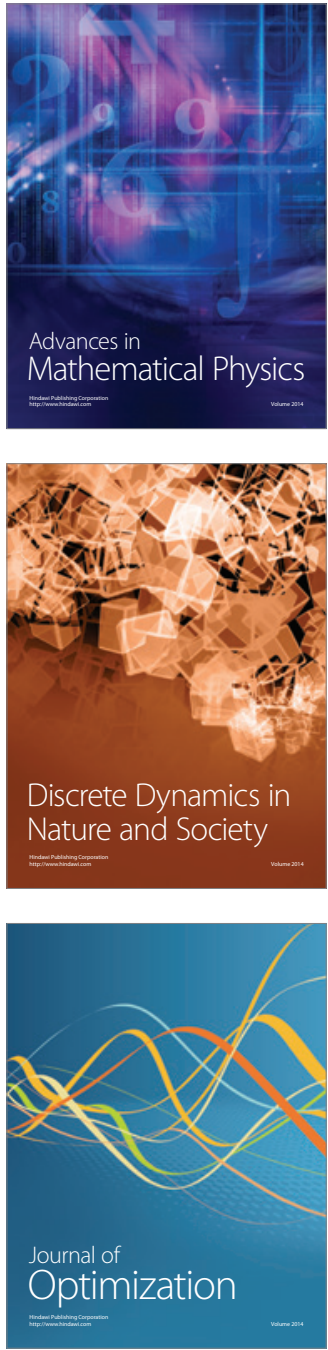